\title{
VIRGO AND OTHER LATE-TYPE DWARFS
}

\author{
N. BROSCH, A. HELLER AND E. ALMOZNINO \\ Wise Observatory $\mathcal{B}$ Dept. of Astronomy and Astrophysics \\ Tel Aviv University, Tel Aviv 69978, Israel
}

We attempt to solve the question of star formation triggers and star formation laws by studying samples of simple objects and defining carefully the possible external effects. Among the star formation (SF) triggers there are some that can operate only in large disk galaxies. These are shear instabilities and density waves, and we can eliminate them if we restrict the sample to diskless objects of low mass. Such galaxies, which do show star formation, are late-type dwarf galaxies (DGs).

Other SF triggers are related to the neighborhood a galaxy finds itself in. Such triggers are galaxy-galaxy collisions and galaxy-intracluster matter interactions (stripping, shocks, etc.). These also can be eliminated by properly choosing the sample to study; one selects galaxies from neighborhoods of widely different densities (of galaxies) and compares their SF parameters.

We selected our first samples in a region of relatively high galaxy density, where a complete morphological classification of objects was available, and suitable depth in brightness sampling could readily be achieved. This is the Virgo cluster, where Binggeli, Sandage and Tammann (1985, BST) provide a sample of more than 2000 DGs. Among those, some $25 \%$ are of late type and are classified by BST as blue compact dwarfs (BCDs), as Magellanic irregulars (Im) of five possible sub-classes, or as combinations of those two classifications.

We study four samples of DGs in the Virgo cluster selected on the basis of average blue surface magnitude as an indicator of recent star formation activity. All galaxies are brighter than $\mathrm{m}_{B}=17.21$. The selection of Virgo late-type DGs carries an additional advantage, as all the objects have total HI measurements from Arecibo by Hoffman et al. $(1987,1989)$. This allows us to consider also the raw material reservoir size's influence on the SF.

Our study relies primarily on CCD surface photometry in BVRI and $\mathrm{H} \alpha$ done at the Wise Observatory (WiseObs). Here we present results of a study of BCDs $(\mathrm{N}=17)$ and low surface brightness (LSB) DGs $(\mathrm{N}=29)$ in Virgo, and explain our plans to study a complementary sample in the extreme field. The BCD sample is the $1996 \mathrm{PhD}$ thesis of EA and the LSB sample is AH's PhD subject.

The integrated photometry of BCDs showed, through a comparison with predictions of evolutionary synthesis programs, that it is possible to understand these systems as resulting from burst evolution of DGs. In general, we found evidence for (at least) one SF burst and an older population, perhaps older than a few $10^{8} \mathrm{yrs}$, which also fits an SF burst model. One object could be the result of a single, recent SF burst with no older population required. We found no significant differences among the high-surface-brightness (HSB) sample galaxies as a function of total HI or other observables. Preliminary results for the low-surface- brightness (LSB) sample indicate a similar lack of correlation with total $\mathrm{HI}$.

The observational data for LSB DGs have been completed in $\mathrm{H} \alpha$, and we present here preliminary results regarding the statistics of HII regions and a comparison with similar parameters for BCDs. The HII regions were detected from "net $\mathrm{H} \alpha$ " images of the galaxies, totalling about 90 minutes of exposure in the line filter. 
We find that the LSB sample shows present star formation activity, in the form of HII regions, in more than $50 \%$ of the sample objects. The sizes of HII regions are similar to those found for the HSB sample. The difference among HSB and LSB galaxies appears to be in the intensity of the starbursting activity and in the relative coverage of the LSB galaxies by HII regions, which is much smaller than for the HSB sample.

We find that whereas for "pure" BCDs $\sim 63 \%$ of their (broad-band delimited) area is covered by HII regions, this fraction drops to only $20 \%$ for the LSBs (it is $30 \%$ among the 'mixed morphology' galaxies). Only a weak positive correlation is revealed between the presence of HII regions among LSBs and the total amount of HI, but this requires further investigation. Finally, we found no dependence of the presence of HII regions in late-type dwarf galaxies of our joined (BCD+LSB) sample with Virgocentric distance. This argues against galaxy interactions being significant triggers of SF.

\section{Acknowledgements}

We acknowledge support by a Center of Excellence grant from the Israel Science Foundation.

\section{References}

Binggeli, B., Sandage, A., and Tammann, G.A. (1985) Astron. J., 90, 1681

Hoffman, G.L., Glosson, J., Helou, G., Salpeter, E.E., and Sandage, A. (1987) Astrophys. J. Suppl., 63, 247

Hoffman, G.L., Williams, H.L., Salpeter, E.E., Sandage, A., and Binggeli, B. (1989) Astrophys. J. Suppl., 71, 701 